\title{
Simulação de um canal rádio UWB através do modelo auto-regressivo
}

\author{
Fábio A. L. da Silva, Leni J. de Matos e Edson Cataldo
}

\begin{abstract}
Resumo - Este artigo apresenta a modelagem e a simulação do canal rádio de banda ultralarga, com linha de visada entre transmissor e receptor, em um ambiente interno (indoor) da Universidade Federal Fluminense, baseado no modelo autoregressivo da função seletividade em freqüência do canal rádio, medida no ambiente. Cinco métodos para implementar modelos auto-regressivos de segunda ordem são propostos, e os resultados da simulação do canal nesses modelos são comparados ao canal real, através dos parâmetros de curta escala do canal (banda de coerência, retardo médio e espalhamento de retardo). Os resultados obtidos são discutidos e, então, ajustes são propostos nos modelos de modo a se obter um melhor modelo do canal.
\end{abstract}

Palavras-chave - simulação, canal rádio UWB, banda ultralarga, modelo auto-regressivo.

Abstract - This paper introduces the modeling and the simulation of the ultra-wideband radio channel, with LOS between transmitter and receiver, in an indoor environment of the Federal Fluminense University, based in the autoregressive model of the frequency selectivity function of the radio channel, measured in the environment. Five methods to implement second order autoregressive models are proposed, and the results of the channel simulation in these models are compared to the real channel, through the small scale channel parameters (coherence bandwidth, mean delay and delay spread). The results obtained are discussed and, then, adjustments are proposed to the model in order to obtain a better model of the channel.

Keywords - simulation, UWB radio channel, ultra-wideband, autoregressive model.

\section{INTRODUÇÃO}

A tecnologia de comunicação em faixa ultralarga (UWB ultra-wideband), cujos sinais têm largura de banda de frequência maior que $20 \%$ da frequência central da banda, tornou-se uma solução importante para a implementação de sistemas de comunicações móveis de altas taxas de transmissão e de curto alcance, como, por exemplo, rede sem fio para dispositivos pessoais, também conhecida por wireless personal area network (WPAN) [1].

Um fator limitante para a operação de um sistema de comunicação sem fio é o canal rádio. Assim, é importante que seja adotado um modelo de canal rádio que reproduza, da melhor forma possível, o canal real para que se possa verificar o desempenho de tais sistemas. Estudos de modelos de canal rádio faixa-larga [2]-[4] serviram como base para que estudos recentes a respeito de modelos para canais rádio UWB fossem desenvolvidos [5]-[7], os quais analisam as propriedades estocásticas da função resposta ao impulso do canal (domínio

Fábio A. L. da Silva, Leni Joaquim de Matos (orientadora) e Edson Cataldo (orientador), Departamento de Engenharia de Telecomunicações, Escola de Engenharia, Universidade Federal Fluminense, Niterói, RJ, E-mails: fadelia@oi.com.br, lenijm@ig.com.bre ecataldo@vm.uff.br. tempo e retardo) ou a função seletividade de frequência do canal (domínio frequiência e tempo).

Este artigo aborda o modelo auto-regressivo (AR) aplicado à função seletividade em frequiência do canal, denotada por $T(f, t)$, por ser uma técnica que requer um pequeno número de parâmetros para modelar o canal rádio [4].

$\mathrm{O}$ modelo AR estima uma determinada função, a partir de alguns valores conhecidos dessa função [8]. No caso da aplicação do modelo (AR), para a simulação de um canal UWB, a função a ser estimada é $T(f, t)$, que é obtida através da sondagem no domínio da freqüência. Esta técnica emprega um analisador de rede vetorial acoplado a duas antenas, uma transmissora (TX) e outra receptora (RX), separadas por uma determinada distância, para executar uma varredura discreta na banda de freqüência a ser analisada.

$\mathrm{O}$ artigo está distribuído da seguinte forma: a Seção II descreve como foram obtidas as medidas do canal rádio a ser simulado; a Seção III apresenta o modelo AR para simulação do canal rádio no domínio da frequiência, a Seção IV trata da modelagem, apresentando os quatros métodos baseados em [4] e o método baseado em [5]; a Seção V mostra os resultados das simulações e a Seção VI, as conclusões.

\section{DESCRIÇÃo DO AMBIENTE DE MEDIDAS}

O ambiente usado foi o hall de entrada do Bloco D da Escola de Engenharia da Universidade Federal Fluminense, como esquematizado na Figura 1.

Os dados foram obtidos por C. F. de Souza, em [9], em sua dissertação de mestrado, de acordo com a configuração descrita a seguir.

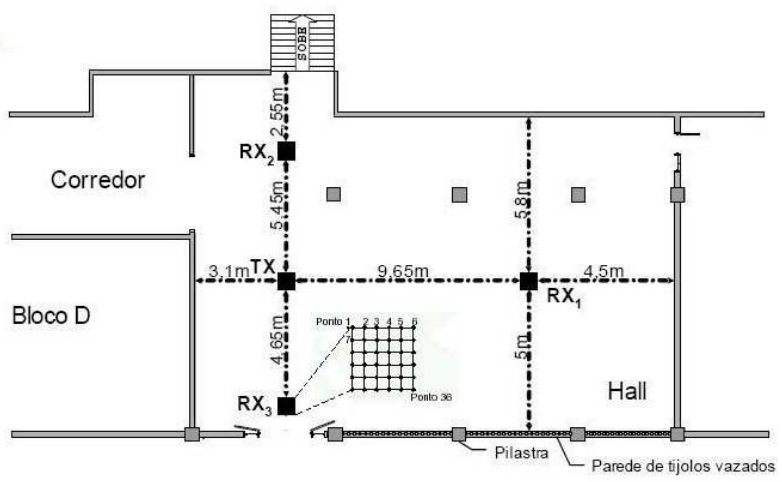

Fig. 1. Planta baixa do ambiente indoor medido.

O transmissor (TX) ficou fixo a uma distância aproximada de 3,1 m da parede do bloco D, enquanto o receptor foi deslocado para três regiões distintas $\left(\mathrm{RX}_{1}, \mathrm{RX}_{2}\right.$ e $\left.\mathrm{RX}_{3}\right)$, com linha de visada (LOS - Line of Sight) para o transmissor. Cada região de 
recepção é formada por um quadrado, de $75 \mathrm{~cm}$ de lado, representado por uma matriz quadrada $(6 \times 6)$, totalizando 36 pontos de medidas de $T(f, t)$. As entradas da matriz estão espaçadas de $15 \mathrm{~cm}$ uma da outra. Foi utilizada a técnica de varredura no domínio da freqüência para as medições, com as características apresentadas na Tabela I.

TABELA I

CARACTERÍSTICAS DA SONDAGEM ADOTADA

\begin{tabular}{|c|c|c|c|}
\hline Banda & $\begin{array}{c}\text { Frequência } \\
\text { central } \\
(\mathrm{MHz})\end{array}$ & $\begin{array}{c}\text { Amostras } \\
\text { por } \\
\text { varredura }\end{array}$ & $\begin{array}{c}\text { Tempo de } \\
\text { varredura } \\
(\mathrm{ms})\end{array}$ \\
\hline \hline 750 & 1335 & 1601 & 696 \\
\hline
\end{tabular}

A sondagem do canal permitiu o levantamento de seus parâmetros de curta escala [10] para cada ponto de medição. Tais parâmetros definem a dispersão temporal do sinal rádio no canal medido. São eles: banda de coerência, retardo médio e espalhamento de retardo.

A banda de coerência representa a menor separação em freqüência para a qual a função de autocorrelação de $T(f, t)$ corresponda, tipicamente, a um nível de $90 \%$ de correlação. Portanto, é a largura de faixa onde as componentes espectrais são afetadas de modo similar.

O retardo médio e o espalhamento de retardo representam, respectivamente, a média e a variância do perfil de potência de retardo normalizado. Tal perfil é obtido através da normalização do quadrado do módulo da transformada inversa de Fourier de $T(f, t)$, no domínio da freqüência.

As funções $T(f, t)$ formam um processo estocástico. Observouse, porém, que cada região de recepção $\left(\mathrm{RX}_{1}\right.$ a $\left.\mathrm{RX}_{3}\right)$ não apresentou significativa variabilidade dentro do curto tempo de varredura da banda de freqüência. Desta forma, assume-se que o processo estocástico é estacionário no sentido amplo e cada função $T(f, t)$ foi medida em um único instante $t$.

Com essas condições, a autocorrelação, $R(k, 0)$, do processo $T(f, t)$, função somente da diferença entre as frequiências, $k$, é calculada por:

$$
R(k)=\frac{1}{N} \sum_{i=1}^{N} T *\left(f_{n}, t\right) . T\left(f_{n-i}, t\right)
$$

onde $N$ é o número de amostras obtidas em uma varredura da banda de frequência e $\mathrm{T}\left(f_{n}, \mathrm{t}\right)$ é o valor medido na frequência discreta $f_{n}$, da banda medida, e $\mathrm{T}^{*}\left(f_{n}, \mathrm{t}\right)$ é o complexo conjugado de $\mathrm{T}\left(f_{n}, \mathrm{t}\right)$.

\section{ModELO AUTO-REGRESSIVO}

De acordo com o modelo AR, o valor de $T\left(f_{n}, t\right)$ é resultado de um processo $\mathrm{AR}$ de ordem $p$, tal que:

$$
T\left(f_{n}, t\right)-\sum_{i=1}^{p} a_{i} T\left(f_{n}-i, t\right)=V\left(f_{n}, t\right)
$$

onde a função $V(f, t)$, resultante da equação (2), é um ruído branco gaussiano complexo, cuja variância é dada por:

$$
\sigma_{V}^{2}=R(0)-\sum_{i=1}^{p} a_{i} \cdot R(i)
$$

e os parâmetros $a_{i}$ da equação (2) são obtidos através da solução da equação de Yule-Walker:

$$
R(-m)-\sum_{i=1}^{p} a_{i} \cdot R(i-m)=0
$$

onde $m \geq 0$ e $p$ é a ordem do modelo AR.

Aplicando-se a transformada $\mathrm{Z}$ ao domínio de $f$ na equação (2), obtém-se pela relação entre $T(f, t)$ e $V(f, t)$ a função de transferência $G(Z)$, que é um filtro linear de $p$ pólos com resposta infinita ao impulso (IIR - infinite impulse response). Assim, o modelo AR é capaz de gerar a função $\hat{T}(f, t)$, função estimada de $T(f, t)$, quando o filtro $G(Z)$ é excitado por um ruído branco gaussiano complexo, de variância dada pela equação (3).

Neste trabalho, a ordem escolhida para o modelo AR foi igual a 2, isto é, $p=2$. Tal escolha foi baseada nos critérios adotados por Howard e Pahlavan [4] e Ghassemzadeh et al. [5], que definiram esse valor para seus respectivos modelos. Outras ordens foram aqui testadas, porém, a pequena melhora nos resultados não compensou o aumento da complexidade do modelo, em conseqüência do aumento da ordem. Nesse caso, a função de transferência do filtro IIR possui dois pólos $\left(p_{1}\right.$ e $\left.p_{2}\right)$ que, juntamente com a variância de $V(f, t)$, são os parâmetros a serem definidos no modelo AR do canal rádio UWB.

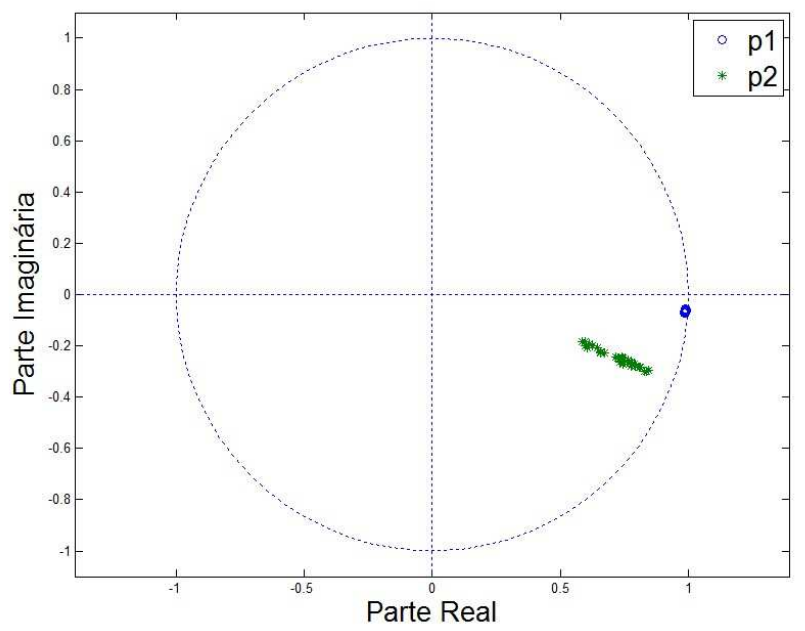

Fig. 2. Disposição no plano $\mathrm{Z}$ dos pólos $p_{1}$ e $p_{l}$, obtidos no processo AR das medidas de $T(f, t)$ em $\mathrm{RX}_{3}$.

O gráfico mostrado na Figura 2 apresenta a disposição, no plano $Z$, dos pólos $p_{l}$ e $p_{2}$, encontrados a partir da aplicação do processo AR para cada uma das 36 funções $T(f, t)$, medidas na matriz da região de recepção $\mathrm{RX}_{3}$. Tais medidas serviram como base para simulação do canal nesta região.

As condições iniciais do filtro $G(Z)$ influenciam na simulação do modelo AR. Conforme mostrado nos gráficos da Figura 3, onde adotou-se o filtro $G(Z)$, resultante do processo AR de $T(f, t)$, medida no ponto 17 da região $\mathrm{RX}_{3}$, para simular o canal a partir da terceira freqüência discreta da banda $\left(f_{3}\right)$.

Comparou-se o referido $T(f, t)$ com as funções $\hat{T}(f, t)$, resultantes da aplicação da função $V(f, t)$, obtida pela equação (2), na entrada de $G(Z)$ com condições iniciais nulas e com 
condições iniciais não nulas. Neste segundo caso, tais condições são os valores $T\left(f_{1}, t\right)$ e $T\left(f_{2}, t\right)$, medidos na primeira $\left(f_{1}\right)$ e na segunda $\left(f_{2}\right)$ freqüências da banda de frequência discreta.

Constatou-se que ambas as funções estimadas apresentam, no início da banda de frequiência, valores diferentes da função medida. A partir da frequência de $1100 \mathrm{MHz}$, a função estimada, com condições iniciais nulas, é aproximadamente igual à função medida. Já a função estimada com condições iniciais, $T\left(f_{1}, t\right)$ e $T\left(f_{2}, t\right)$, aproxima-se da função medida, em uma frequência posterior, a partir da freqüência de $1400 \mathrm{MHz}$.

Dessa forma, para este trabalho, foram adotadas condições iniciais nulas para o filtro IIR, do modelo $\mathrm{AR}$, e o canal rádio foi modelado na faixa de 1100 a $1750 \mathrm{MHz}$ na qual a função $\hat{T}(f, t)$ aproxima-se da função $T(f, t)$.

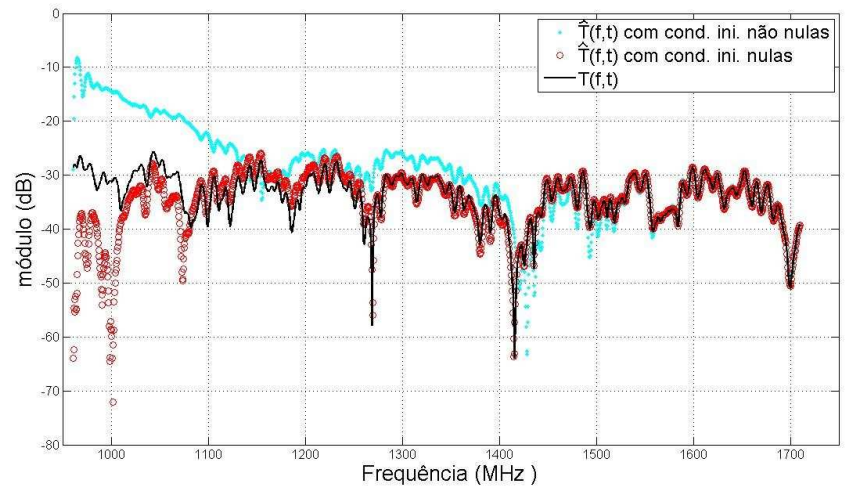

(a)

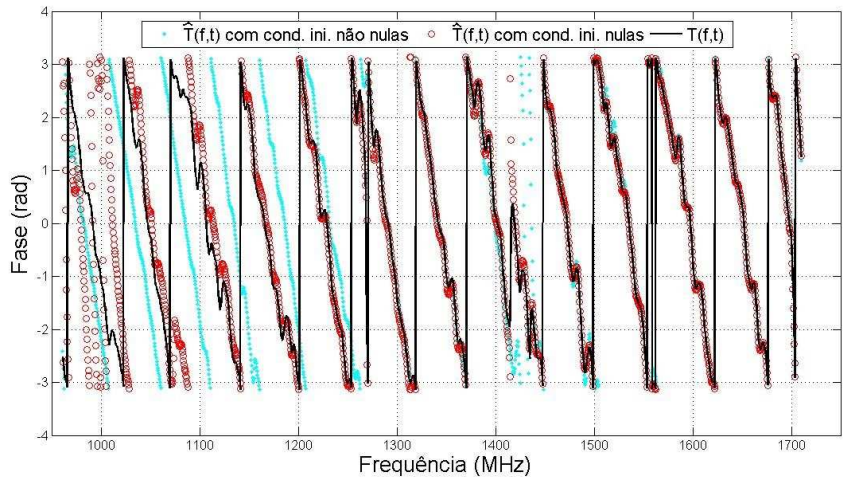

(b)

Fig. 3. Comparação entre $T(f, t)$ medida no ponto 17 de $\mathrm{RX}_{3}, \hat{T}(f, t)$ gerada com condições iniciais não nulas e $\hat{T}(f, t)$ gerada com condições iniciais nulas (a) resposta, em freqüência, do módulo e (b) resposta, em freqüência, da fase.

\section{ModelageM}

Para modelar um canal rádio, baseado no processo AR de $T(f, t)$, é necessário especificar um modelo para o módulo e a fase dos pólos do modelo AR e para a variância de $V(f, t)$, que está relacionada com a potência média recebida no ponto de medida. Embora, na realidade, esse modelo deva ser completamente probabilístico, em alguns casos a aproximação por um modelo determinístico pode ser usada, devido às características dos sinais medidos. A seguir, serão discutidos cinco métodos para a modelagem do canal, partindo dos dados de módulo e fase dos pólos do modelo AR. Os métodos de (i) a (iv) foram especificados de maneira semelhante ao que foi empregado em [4] e o método (v) foi baseado nas especificações determinadas por [5].

Para os métodos de (i) a (iv), a fase de $p_{1}$ é fixa e igual à média das fases dos pólos $p_{l}$ e as demais características dos cinco métodos são descritos a seguir:

- (i) os módulos de $p_{1}$ e $p_{2}$ e a fase de $p_{2}$ são variáveis aleatórias gaussianas, de média e variância obtidas através da distribuição dos respectivos pólos no plano Z;

- (ii) a fase de $p_{2}$ é a média das fases dos pólos $p_{2}$ e os módulos de $p_{1}$ e $p_{2}$ são variáveis aleatórias gaussianas como em (i);

- (iii) o módulo e a fase de $p_{2}$ são iguais aos valores médios de módulo e da fase dos pólos $p_{2}$ e o módulo de $p_{I}$ é uma variável aleatória gaussiana como em (i);

- (iv) os módulos de $p_{1}$ e $p_{2}$ e a fase de $p_{2}$ são iguais às médias das distribuições dos respectivos pólos no plano $\mathrm{Z}$; e

- (v) os módulos de $p_{1}$ e $p_{2}$ são variáveis aleatórias com distribuição Weibull e as fases de $p_{1}$ e $p_{2}$ são variáveis aleatórias gaussianas.

Assumiu-se, para todos os métodos, que a variância do ruído branco, entrada do filtro $G(Z)$, é igual a:

$$
\sigma_{V}^{2}=\frac{R(0)}{g(n) \otimes g *(-n)}, n=0
$$

onde $R(0)$ representa a potência média recebida num dado ponto da região $\mathrm{RX}_{3}$, que é modelada como uma função lognormal, de média e variância obtidas por intermédio das 36 medidas de $T(f, t)$, na referida região, e $g(n)$ é a resposta ao impulso do filtro IIR do modelo AR construído por cada método.

De acordo com [4], o modelo para potência média recebida, que foi adotado neste estudo, é adequado para modelar o canal rádio em uma pequena área, como a região $\mathrm{RX}_{3}$. Entretanto, é possível gerar um modelo AR capaz de simular $T(f, t)$ em função da distância TX-RX, se for adotado um modelo para potência média recebida mais complexo, como em [5], que represente a atenuação do sinal em função da distância.

\section{SimulaçõES E RESUltados}

Após gerar 100 funções $\hat{T}(f, t)$, para cada um dos cinco métodos utilizados, foi feita a comparação dos parâmetros de curta escala: banda de coerência, retardo médio e espalhamento de retardo. Esses parâmetros foram obtidos tanto para as funções $\hat{T}(f, t)$ como para as funções $T(f, t)$.

As Figuras 4, 5 e 6 mostram, respectivamente, comparações entre os gráficos das funções distribuições de probabilidade da banda de coerência, retardo médio e espalhamento de retardo, para o canal medido e para o canal simulado, através do emprego dos cinco métodos descritos anteriormente.

Observa-se, nestes gráficos, que a faixa de variação dos parâmetros do canal rádio medido está contida na faixa de variação dos parâmetros do canal simulado pelos cinco métodos adotados. Portanto, todos os métodos empregados foram capazes de gerar modelos compatíveis ao canal rádio. 


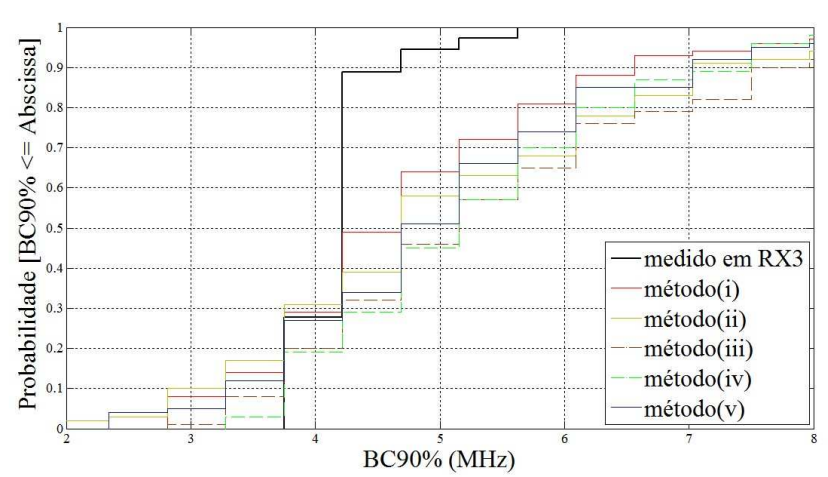

Fig. 4. Função distribuição de probabilidade para a banda de coerência de $90 \%$ (BC90\%) dos canais simulados e medido.

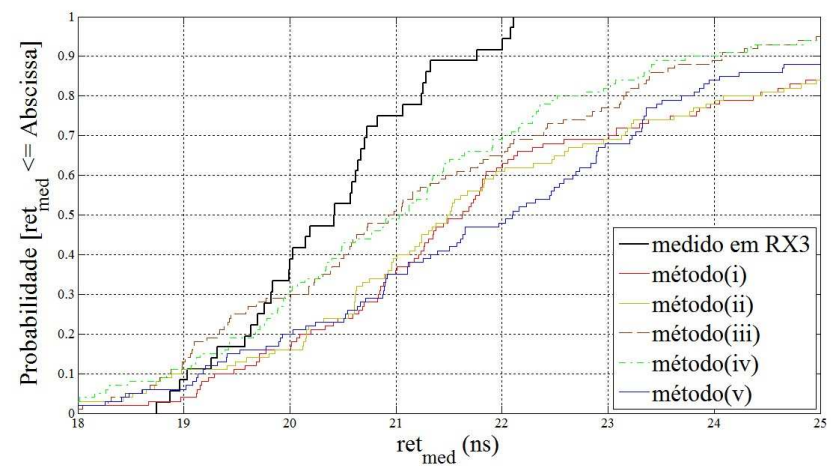

Fig. 5. Função distribuição de probabilidade para o retardo médio (ret $\left.{ }_{\text {med }}\right)$ dos canais simulados e medido.

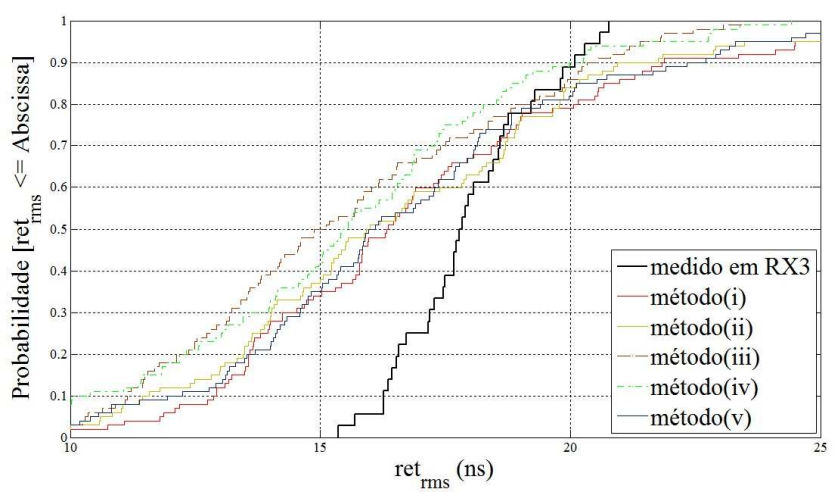

Fig. 6. Função distribuição de probabilidade para o espalhamento de retardo $\left(\right.$ ret $\left._{\mathrm{rms}}\right)$ dos canais simulados e medido.

Outra ferramenta ainda utilizada, para comparar o canal rádio simulado com o canal real, foi verificar se a autocorrelação $R(k)$ das 36 funções $T(f, t)$ encontram-se dentro do intervalo máximo e mínimo da autocorrelação, $\hat{R}(k)$, das funções $\hat{T}(f, t)$, ou seja, no intervalo de confiança da autocorrelação. Como exemplo, foram geradas 500 funções $\hat{T}(f, t)$ pelo método (iv), o menos complexo entre os cinco apresentados. Então, verificou-se, conforme mostrado na Figura 7, que os módulos das autocorrelações das 36 funções $T(f, t)$, da região $\mathrm{RX}_{3}$, encontram-se no interior do intervalo das autocorrelações, $\hat{R}(k)$, das funções $\hat{T}(f, t)$. Portanto, este método pode gerar funções $\hat{T}(f, t)$ que apresentam autocorrelações compatíveis às autocorrelações das funções $T(f, t)$.

Várias outras ferramentas poderiam ter sido usadas, partindo do mesmo princípio, como usar outros métodos. Além disso, seria necessário um estudo de convergência para determinar o número de funções $\hat{T}(f, t)$ que deveriam ser geradas. Porém, o objetivo aqui, é apenas mostrar a possibilidade de mais uma ferramenta a ser utilizada para possibilitar a comparação entre os canais real e simulado que, nesse caso, mostrou um ótimo comportamento.

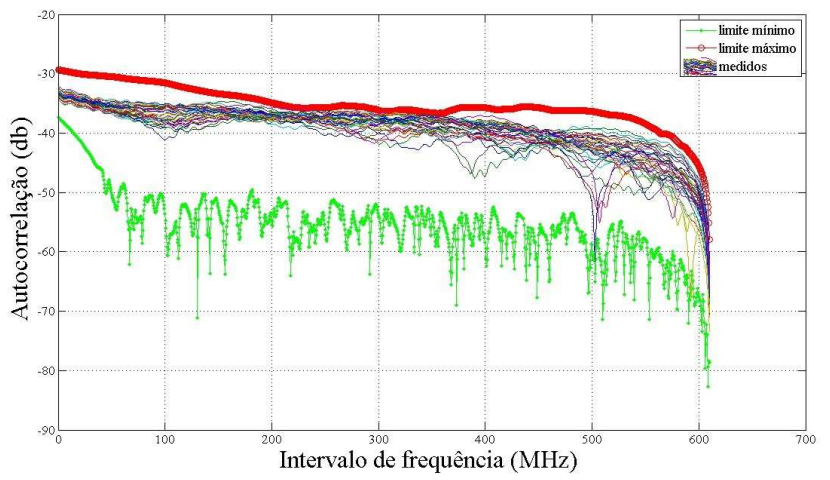

Fig. 7. Autocorrelação das 36 funções medidas em $\mathrm{RX}_{3}$, limite máximo e limite mínimo da autocorrelação das funções simuladas pelo método (iv).

\section{CONCLUSÕES}

A modelagem do canal rádio, em um ambiente indoor escolhido, foi proposta e os resultados simulados foram comparados com os dados experimentais. A modelagem proposta foi baseada em trabalhos anteriores, porém ajustes, em termos de condições iniciais do filtro e faixa de variação dos pólos $p_{1}$ e $p_{2}$, foram realizados a fim de adequar o modelo AR aos resultados medidos disponíveis.

Todos os cinco métodos empregados foram capazes de gerar funções estimadas $\hat{T}(f, t)$ que apresentaram mesmas características das funções $T(f, t)$ medidas, entretanto, em termos de complexidade computacional, o método (iv) mostrou-se mais adequado uma vez que foi gerado um modelo AR de pólos determinísticos.

Logo, o uso do modelo AR, com os devidos ajustes, como diminuir a banda de freqüência simulada em relação à banda medida, mostrou-se adequado para modelar o canal rádio, com baixa complexidade e pequena quantidade de parâmetros.

Objetivando melhorar a modelagem do canal, ajustes ainda podem ser feitos, destacando-se dentre eles:

- empregar um número maior de medidas de $T(f, t)$ e um modelo de atenuação do sinal com a distância, a fim de aumentar a complexidade do ambiente e obter uma faixa maior de valores para os parâmetros do modelo;

- propor outras funções distribuições de probabilidade para os pólos do modelo AR, empregando o Princípio da Máxima Entropia [11] na determinação dessas distribuições de 
probabilidade, uma vez que esse Princípio é bem aplicado nesse caso, pois a quantidade de dados experimentais é pequena;

- discutir o problema gerado pela resposta inicial do filtro IIR, impedindo que a função $T(f, t)$ seja estimada em toda a banda de frequência medida, nesse caso, duas direções podem ser tomadas: detectar as condições iniciais do filtro que ajustem melhor a resposta inicial ou empregar um modelo AR modificado.

\section{REFERÊNCIAS}

[1] G. R. Aiello e G. D. Rogerson, "Ultra-wideband Wireless Systems", IEEE Microwave Magazine, v. 4, pp. 36-47, Junho 2003.

[2] A. A. M. Saleh e R. A. Valenzuela, "A statistical model for indoor multipath propagation”, IEEE J. Select. Areas Commun., v. 5, pp. 128-137, Fevereiro 1987.

[3] H. Hashemi, "The indoor radio propagation channel", Proc. IEEE, v. 81, pp. 943-968, Julho 1993.

[4] S. Horward e K. Pahlavan, "Autoregressive Modeling of Wide-Band Indoor Radio Propagation", IEEE Transaction on Commun., v. 40, pp. 1540-1552, Setembro 1992.

[5] S. S. Ghassemzadeh, R. Jana,V. Tarokh, C. W. Rice e W. Turin, "Measurement and Modeling of an Ultra-Wide Bandwidth Indoor Channel", IEEE Transactions on Comm., v. 52, pp. 1786-1796, Outubro 2004.

[6] D. Cassioli, M. Z. Win e A. Molisch, "The ultra-wide bandwidth indoor channel: from statistical model to simulations", IEEE J. Select. Areas Commun., v. 20, n. 6, pp. 1247-1257, Agosto 2002.

[7] A. F. Molisch, J. R. Foerster e M. Pendergrass, "Channel models for ultra-wideband personal area networks", IEEE Wireless Commun. Mag., v. 10, n. 6, pp. 14-21, Dezembro 2003.

[8] S. Haykin, Adaptive Filter Theory, Upper Saddle River, NJ:Pertince-Hall,1996.

[9] C. F. de Souza, Análise da dispersão temporal de canais de banda ultralarga (UWB) através de medidas realizadas em ambientes internos e externos, Dissertação de Mestrado, Universidade Federal Fluminense, Niterói, 2006.

[10] D. Parsons, The Mobile Radio Propagation Channel, New York: John Wiley \& Sons, 1992.

[11] J. N. Kaput e H. K. Kesavan, Entropy optimization principles with applications, Academic Press Inc., 1992. 